\title{
Work Smart conference: A collaboration by Royal College of Physicians chief registrars in Wessex
}

\author{
Author: Susan Hayward
}

Aim

To provide an opportunity help trainees better understand the need and value of wellbeing.

\section{Method}

As a team in Wessex, the chief registrars agreed there was potential benefit to providing a conference for other trainee doctors on wellbeing. Due to the number of chief registrars within the region, this was an excellent opportunity for a collaborative approach.

A date was chosen using a Doodle poll, ideas were brainstormed using a WhatsApp group, a suitable venue was chosen and booked, and some funding was negotiated from Southampton University. With the number of people involved, there was a large resource of ideas and contacts for speakers and workshops.

At the first planning meeting the initial discussion allowed us to assess our aim and provided rapid 'plan, do, study, act' cycles to discuss options. It was felt this should be trainee-led with little senior presence in order to allow open discussions.

\section{Results}

The event is booked for mid-May. Data will be available from before and after regarding current knowledge and actions around wellbeing.

The sessions will include: What we can learn from being an athlete; getting the best out of family life; what you can learn from kindness, yoga, gym or swim sessions; nutrition and sleep advice; mindfulness; and how to facilitate change in the workplace.

\section{Conclusion}

The benefits to having multiple chief registrars in a region include the opportunity for collaborative working. The learning we expect to take place from this conference is targeted at the need we have discovered in our investigations at trusts. The experience from this conference may create opportunities for further events of this nature.

Author: Royal College of Physicians chief registrar, Salisbury NHS Foundation Trust, UK

\section{Conflict of interest statement}

None. 